\title{
AN EFFECTIVE BOUND FOR REFLEXIVE SHEAVES ON CANONICALLY TRIVIAL 3-FOLDS
}

\author{
PETER VERMEIRE
}

\begin{abstract}
We give effective bounds for the third Chern class of a semistable rank 2 reflexive sheaf on a canonically trivial threefold.
\end{abstract}

\section{INTRODUCTION}

We work over an algebraically closed field of characteristic 0 .

In a previous work [5] we showed that if $\mathcal{F}$ is a semistable rank 2 reflexive sheaf on a smooth projective threefold $X$ with $\operatorname{Pic}(X)=\mathbb{Z}$, then there is an upper bound on $c_{3}(\mathcal{F})$ in terms of $c_{i}(X), c_{1}(\mathcal{F})$ and $c_{2}(\mathcal{F})$ (note that $c_{3}(\mathcal{F}) \geq 0$ ). It has more recently been conjectured [2] that this remains true for any smooth, projective threefold. Here we derive explicit effective bounds in the case of a polarized smooth projective threefold $X$ with $\omega_{X}=\mathcal{O}_{X}$, without the restriction on the Picard group.

\section{Stability And Boundedness}

Definition 1. Let $L$ be a very ample line bundle on a smooth projective variety $X$. A rank 2 reflexive coherent sheaf $\mathcal{F}$ on $X$ is $\boldsymbol{L}$-stable (resp. $\boldsymbol{L}$-semistable) if for every invertible subsheaf $\mathcal{F}^{\prime}$ of $\mathcal{F}$ with $0<\operatorname{rank} \mathcal{F}^{\prime}<\operatorname{rank} \mathcal{F}$, we have $\mu\left(\mathcal{F}^{\prime}, L\right)<\mu(\mathcal{F}, L)($ resp. $\leq)$, where

$$
\mu(\mathcal{F}, L)=\frac{c_{1}(\mathcal{F}) \cdot[L]^{\operatorname{dim} X-1}}{(\operatorname{rank} \mathcal{F})[L]^{\operatorname{dim} X}}
$$

Definition 2. A reflexive sheaf $\mathcal{F}$ is normalized with respect to $\boldsymbol{L}$ if $-1<\mu(\mathcal{F}, L) \leq 0$. As $L$ is typically fixed, we usually say simply that $\mathcal{F}$ is normalized. Note that as $\mu(\mathcal{F} \otimes L, L)=\mu(\mathcal{F}, L)+1$, there exists, for any fixed $\mathcal{F}$, a unique $k \in \mathbb{Z}$ such that $\mathcal{F} \otimes L^{k}$ is normalized with respect to $L$.

Date: December 3, 2013.

2010 Mathematics Subject Classification. 14J60, 14J32. 
For a fixed smooth, canonically trivial $X \subset \mathbb{P}^{n}$, our goal is to give a bound on $c_{3}(\mathcal{F})$ in terms of $c_{1}(\mathcal{F})$ and $c_{2}(\mathcal{F})$.

Lemma 3. Let $X$ be a smooth projective canonically trivial threefold, $L$ any very ample line bundle on $X, \mathcal{F}$ a rank two reflexive sheaf. If $\mathcal{F}$ admits a section $s \in \Gamma(\mathcal{F})$ whose zero locus is a curve $Y$, then

$$
c_{3}(\mathcal{F}) \leq d^{2}-3 d-c_{1}(\mathcal{F}) c_{2}(\mathcal{F})
$$

where $d=c_{1}(L) c_{2}(\mathcal{F})$ is the degree of $Y$ in the embedding induced by $L$.

Proof: This is directly related to the Hartshorne-Serre correspondence [3, Theorem 4.1]. It is shown there that any such $Y$ is locally Cohen-Macaulay, and it can be immediately deduced [5, Theorem 1] that $c_{3}(\mathcal{F})=2 p_{a}(Y)-2-c_{2}(\mathcal{F}) c_{1}\left(\omega_{X}\right)-c_{1}(\mathcal{F}) c_{2}(\mathcal{F})$. In the canonically trivial case $c_{2}(\mathcal{F}) c_{1}\left(\omega_{X}\right)=0$, and in any case the degree of the curve section $Y$ in the embedding given by $L$ is $d=c_{1}(L) c_{2}(\mathcal{F})$. The fact that $2 p_{a}(Y)-2 \leq d^{2}-3 d$ is the bound coming from the degree of a plane curve.

In some common situations, we can do better:

Proposition 4. Let $X$ be a smooth projective canonically trivial threefold, $L$ a very ample line bundle, $\mathcal{F}$ a stable (resp. semistable) rank two reflexive sheaf. Suppose that $\mu(\mathcal{F}, L) \geq 1$ (resp. $\mu(\mathcal{F}, L)>1$ ) and that $H^{1}\left(X, \operatorname{det} \mathcal{F}^{*} \otimes L\right)=0$. If $s \in \Gamma(\mathcal{F})$ is a section whose zero locus is a smooth curve $Y$, then

$$
c_{3}(\mathcal{F}) \leq m(m-1)\left(h^{0}(X, L)-2\right)+2 m \epsilon-2-c_{1}(\mathcal{F}) c_{2}(\mathcal{F})
$$

where

$$
\begin{gathered}
m=\left\lfloor\frac{c_{1}(L) c_{2}(\mathcal{F})-1}{h^{0}(X, L)-2}\right\rfloor \\
\epsilon=c_{1}(L) c_{2}(\mathcal{F})-1-m\left(h^{0}(X, L)-2\right)
\end{gathered}
$$

Proof: The section gives the sequence

$$
0 \rightarrow \operatorname{det} \mathcal{F}^{*} \otimes L \rightarrow \mathcal{F}^{*} \otimes L \rightarrow \mathcal{J}_{Y} \otimes L \rightarrow 0
$$

By hypothesis, $\mu\left(\mathcal{F}^{*} \otimes L, L\right) \leq 0$ when $\mathcal{F}$ is stable and $\mu\left(\mathcal{F}^{*} \otimes L, L\right)<$ 0 when $\mathcal{F}$ is semistable. In either case $H^{0}\left(X, \mathcal{F}^{*} \otimes L\right)=0$, and so $H^{0}\left(X, \mathcal{J}_{Y} \otimes L\right)=0$. This implies that $Y$ is a non-degenerate curve in the embedding induced by $L$, hence we may apply Castelnuovo's Bound [1, p. 116].

The idea now is: given a very ample line bundle $L$, bound the twist of $\mathcal{F}$ by $L^{r}$ needed to produce a section, and then use the bound in 
Lemma 3. We do this by first finding a bound for the vanishing of $h^{2}\left(\mathcal{F} \otimes L^{r}\right)$ and then by making the Euler characteristic positive.

The next two results (Proposition 5 and Corollary 7) follow directly from more general results in [5]. The first proceeds by showing that a minimal $m$ satisfying the given inequality but contradicting the claim must be positive; the second proceeds from the first by Serre Duality on the smooth surface $D$, and then the stated inequality forces the Euler characteristic to be positive via Riemann-Roch, hence the relevant $h^{0}$ must be strictly greater than $h^{1}$, and in particular must be positive.

Proposition 5. [5, Proposition 9] Let $X$ be a smooth, projective canonically trivial threefold with very ample line bundle $L, \mathcal{F}$ a normalized $L$-semistable rank 2 reflexive sheaf. Let $D$ be a general member of the linear system $|L|$. Suppose $m<0$ is an integer satisfying

$$
m+\mu(\mathcal{F}, L)+1<0
$$

Then $H^{0}\left(D, \mathcal{F}_{D}(m D)\right)=0$.

Remark 6 Note that the reference contains the hypothesis $\operatorname{Pic}(X)=$ $\mathbb{Z}$, but it is not relevant for this particular proposition.

Corollary 7. [5, Corollary 11] With notation and hypotheses as in Proposition 5, if

$$
r>2+\mu(\mathcal{F}, L)
$$

then for the general member $D, H^{2}\left(D, \mathcal{F}_{D}^{*}(r D)\right)=0$. If, furthermore, $r$ is such that:

$$
\left(6 r^{2}-6 r+2\right)-2(6 r-3) \mu(\mathcal{F}, L) \geq \frac{\left(6 c_{2}(\mathcal{F})-3 c_{1}(\mathcal{F})^{2}-c_{2}(X)\right)[L]}{[L]^{3}}
$$

then $H^{0}\left(D, \mathcal{F}_{D}^{*}(r D)\right) \neq 0$.

Corollary 8. With notation and hypotheses as in Proposition 5 there exists a constant $\rho_{1}$ depending on $L, c_{1}(\mathcal{F}), c_{2}(\mathcal{F})$ and $c_{2}(X)$ such that if $r \geq \rho_{1}$ then $H^{1}\left(D, \mathcal{F}_{D}^{*}(r D)\right)=0$.

\section{ProOF:}

By the previous Corollary, there is a constant depending on the above parameters such that if $k$ is larger than that constant, then $\mathcal{F}_{D}^{*}(k D)$ has a section. Choosing the smallest such integer $k$ we have a sequence:

$$
0 \rightarrow \mathcal{O}_{D} \rightarrow \mathcal{F}_{D}^{*}(k D) \rightarrow \mathcal{J}_{Z}(2 k D) \otimes \operatorname{det} \mathcal{F}^{*} \rightarrow 0
$$

where $Z \subset D$ is zero dimensional of length

$$
\ell=c_{1}(L) c_{2}(\mathcal{F})-k c_{1}^{2}(L) c_{1}(\mathcal{F})+k^{2} c_{1}^{3}(L)
$$


Because $K_{D}=L \otimes \mathcal{O}_{D}$ is (very) ample, $H^{1}(D, \mathcal{O}(D))=H^{2}\left(X, \mathcal{O}_{X}\right)$ and $H^{1}(D, \mathcal{O}(2 D))=0$.

Let $\alpha \in \mathbb{Z}$ be such that $\left|\operatorname{det} \mathcal{F}^{*} \otimes L^{\alpha} \otimes \mathcal{O}_{D}\right|$ contains a curve that misses $Z$ (e.g. if the system is very ample). Because $\mathcal{O}_{D}(D)$ is very ample, we have $H^{1}\left(D, \mathcal{J}_{Z}((2 k+t) D)\right)=0$ when $2 k+t \geq \ell-1$ by a uniform regularity result of Mumford [4, p. 103].

Therefore, by choosing a curve that misses $Z$ in the system $\mid \operatorname{det} \mathcal{F}^{*} \otimes$ $L^{\alpha} \otimes \mathcal{O}_{D} \mid$, we find $H^{1}\left(D, \mathcal{J}_{Z}((2 k+t+\alpha) D) \otimes \operatorname{det} \mathcal{F}^{*}\right)=0$ when $2 k+t+\alpha \geq$ $\ell-1$ and $2 k+t \geq 2$.

Consequently, $H^{1}\left(D, \mathcal{F}_{D}^{*}(r D)\right)=0$ for

$$
r \geq \max \{k+2, \ell-1-k, 2-k+\alpha\}
$$

Corollary 9. With notation and hypotheses as in Proposition 5, there exists an integer $\rho_{2}$ depending on $L, c_{1}(\mathcal{F}), c_{2}(\mathcal{F})$ and $c_{2}(X)$ such that if $r \geq \rho_{2}$ then $H^{0}\left(X, \mathcal{F}^{*} \otimes L^{r}\right) \neq 0$.

Proof: The vanishing of $H^{1}$ and $H^{2}$ on $D$ described in the above Corollaries gives $H^{2}\left(X, \mathcal{F}^{*} \otimes L^{r}\right)=0$. The result now follows by an Euler characteristic argument.

Theorem 10. Let $X$ be a smooth, projective canonically trivial threefold with very ample line bundle $L$, and $\mathcal{F}$ an $L$-semistable rank 2 reflexive sheaf. Then there exists an integer $C$ depending on $L, c_{1}(\mathcal{F}), c_{2}(\mathcal{F})$ and $c_{2}(X)$ such that $C \geq c_{3}(\mathcal{F})$.

ProOF: As $c_{3}(\mathcal{F})$ is unaffected by twisting by a line bundle, we may assume $\mathcal{F}$ is normalized. Then the above results apply and we can take a section of $\mathcal{F}^{*} \otimes L^{k}$ for some $k$, bounded as in Corollary 9. We then have an exact sequence

$$
0 \rightarrow \mathcal{O}_{X} \rightarrow \mathcal{F}^{*} \otimes L^{k} \rightarrow \mathcal{J}_{Y} \otimes L^{2 k} \otimes \operatorname{det} \mathcal{F}^{*} \rightarrow 0
$$

where $Y \subset X$ is a curve. Computing Euler characteristics gives:

$$
2 p_{a}(Y)-2=d_{1} d_{2}+c_{3}(\mathcal{F})
$$

where

$$
d_{1}=c_{1}\left(\mathcal{F}^{*} \otimes L^{k}\right)=-c_{1}(\mathcal{F})+2 k c_{1}(L)
$$

and

$$
\begin{aligned}
d_{2} & =c_{2}\left(\mathcal{F}^{*} \otimes L^{k}\right) \\
& =c_{2}(\mathcal{F})-k c_{1}(\mathcal{F}) c_{1}(L)+k^{2} c_{1}^{2}(L)
\end{aligned}
$$


Note that in the embedding determined by $L$, the degree of the curve $Y$ is precisely $d_{2} c_{1}(L)$. This implies $d_{2} c_{1}(L)\left(d_{2} c_{1}(L)-3\right) \geq 2 p_{a}(Y)-2$ and so

$$
d_{2} c_{1}(L)\left(d_{2} c_{1}(L)-3\right)-d_{1} d_{2} \geq c_{3}(\mathcal{F})
$$

The following is a straightforward calculation as in [5]. For notational convenience we let $d=c_{1}(L) c_{2}(\mathcal{F})$.

Corollary 11. Let $X \subset \mathbb{P}^{4}$ be a smooth quintic hypersurface. Let $\mathcal{F}$ be a rank two semistable reflexive sheaf with $\mu\left(\mathcal{F}, \mathcal{O}_{X}(1)\right)=0$. Then for $d \leq 40$ we have

$$
c_{3}(\mathcal{F}) \leq\left(5 d^{2}+136 d+910\right)\left(5 d^{2}+134 d+880\right) .
$$

Proof: We let $L=\mathcal{O}_{X}(1)$ and note that $[L]^{3}=5$ and $c_{2}(X)=$ $10 c_{1}(L)^{2}$. The first bound in Corollary 7 is $r \geq 3$ and the second becomes $6 r^{2}-6 r+2 \geq \frac{6 d-50}{5}$, or

$$
r \geq \frac{5+\sqrt{20 d-175}}{10}
$$

As long as $d \leq 40$, the second inequality is weaker than the first, hence we need only take $r \geq 3$. In Corollary 8 , we have $\alpha=2$ and $\ell=d-5 r+5 r^{2}$, and hence the bound there becomes $\rho_{1} \geq \max \{r+$ $\left.2,5 r^{2}-6 r+d-1,4-r\right\}$, but the second terms is strongest and we have $\rho_{1} \geq d+14$.

Turning to Corollary 9 , we need $\chi\left(\mathcal{F}^{*} \otimes L^{\rho_{2}}\right)>0$, but Riemann-Roch gives

$$
\chi\left(\mathcal{F}^{*} \otimes L^{\rho_{2}}\right)-\frac{1}{2} c_{3}(\mathcal{F})=\frac{\rho_{2}}{3}\left(5 \rho_{2}^{2}+25-3 d\right)
$$

and this is clearly positive for $\rho_{2} \geq d+14$.

Finally, from Theorem 10 we have $d_{1}=\left(2 \rho_{2}-1\right) c_{1}(L)$ and $d_{2}=$ $c_{2}(\mathcal{F})+\left(\rho_{2}^{2}-\rho_{2}\right) c_{1}^{2}(L)$, and hence the bound for $c_{3}$ becomes

$$
\begin{aligned}
c_{3}(\mathcal{F}) & \leq d_{2} c_{1}(L)\left(d_{2} c_{1}(L)-3\right)-d_{1} d_{2} \\
& =\left(d+5\left(\rho_{2}^{2}-\rho_{2}\right)\right)\left(d+5\left(\rho_{2}^{2}-\rho_{2}\right)-3\right)-\left(2 \rho_{2}-1\right)\left(d+5\left(\rho_{2}^{2}-\rho_{2}\right)\right)
\end{aligned}
$$

Substituting $\rho_{2}=d+14$ yields $c_{3}(\mathcal{F}) \leq\left(5 d^{2}+136 d+910\right)\left(5 d^{2}+134 d+\right.$ 880).

Remark 12 Note that this is somewhat better than the bound found in [5], though that bound was also quartic in $d$. Note that for $\mathbb{P}^{3}, c_{3}(\mathcal{F})$ is bounded by a quadratic in $d[3,8.2]$.

For the next result, recall $[6$, Notation 6$]$ that $\Delta(\mathcal{F})=c_{2}\left(\mathcal{F} \otimes \mathcal{F}^{*}\right)=$ $4 c_{2}(\mathcal{F})-c_{1}^{2}(\mathcal{F})$. 
Proposition 13. Let $X$ be a smooth projective Fano threefold. If $\mathcal{F}$ is a rank 2 semistable reflexive sheaf, then

$$
c_{1}(X) \Delta(\mathcal{F}) \geq \frac{c_{1}(X) c_{2}(X)}{3}-2 .
$$

Proof: [6, Corollary 12] gives

$$
c_{1}(X) \Delta(\mathcal{F})=2 \operatorname{ext}_{\mathcal{O}_{X}}^{1}(\mathcal{F}, \mathcal{F})-2 \operatorname{ext}_{\mathcal{O}_{X}}^{2}(\mathcal{F}, \mathcal{F})+\frac{c_{1}(X) c_{2}(X)}{3}-2
$$

Further, [6, Corollary 15] states that the relevant moduli space of sheaves admits a perfect tangent-obstruction complex and has a virtual cycle of the expected dimension $\operatorname{ext}_{\mathcal{O}_{X}}^{1}(\mathcal{F}, \mathcal{F})-\operatorname{ext}_{\mathcal{O}_{X}}^{2}(\mathcal{F}, \mathcal{F})$. In particular, this expression is non-negative.

As a corollary, we have:

Corollary 14. Let $X \subset \mathbb{P}^{4}$ be a smooth Fano hypersurface of degree $r, \mathcal{F}$ a rank 2 semistable reflexive sheaf with $\mu\left(\mathcal{F}, \mathcal{O}_{X}(1)\right)=0$.

(1) If $r=1,2,3$ then $c_{1}(L) c_{2}(\mathcal{F}) \geq 1$.

(2) If $r=4$ then $c_{1}(L) c_{2}(\mathcal{F}) \geq 0$.

Proof: These follow from (1) by direct computation.

Remark 15 Note that the case $r=1$ is related to well-known bound on $\mathbb{P}^{3}[3,3.3]$; we do not know if these bounds are the best possible.

Acknowledgments: I would like to thank Martin Kool for making me aware of his joint work with Amin Gholampour, and also an anonymous referee for several suggestions that greatly improved the readability of this paper.

\section{REFERENCES}

[1] E. Arbarello, M. Cornalba, P. A. Griffiths, and J. Harris, Geometry of Algebraic Curves, Vol. I, Springer-Verlag, New York, 1985.

[2] A. Gholampour and M. Kool, Stable reflexive sheaves and localization, arXiv:1308.3688.

[3] Robin Hartshorne, Stable Reflexive Sheaves, Math. Ann. 254 (1980): 121-176.

[4] David Mumford, Lectures on Curves on an Algebraic Surface, Princeton University Press, 1966.

[5] P. Vermeire, Stable Reflexive Sheaves on Smooth Projective 3-Folds, Pacific J. Math. 219 (2005), no.2: 391-398.

[6] P. Vermeire, Moduli of Reflexive Sheaves on Smooth Projective 3-folds, Journal of Pure and Applied Algebra 211 (2007), no. 3, pp. 622-632. 
Department of Mathematics, Central Michigan University, Mount Pleasant MI 48859

E-mail address: p.vermeire@cmich.edu 